\title{
Interferometer system for Keda Torus eXperiment using terahertz solid-state diode sources
}

\author{
Wenzhe Mao ${ }^{1}$, Jinlin Xie ${ }^{1}$, Weixing Ding ${ }^{1,2}$, Hong $i^{1}$, Tao Lan ${ }^{1}$, Adi Liu ${ }^{1}$, Wandong Liu ${ }^{1}$ \\ ${ }^{1}$ School of Physics, University of Science and Technology of China, Hefei, Anhui 230026, China, jlxie@ustc.edu.cn \\ ${ }^{2}$ University of California-Los Angeles, P.O.Box 957099, Los Angeles, California 90095-7099, USA
}

A one chord interferometer system based on terahertz solid state source has been first applied on a reversed field pinch (RFP) device, Keda Torus eXperiment (KTX) [1]. The compact and lightweight platform made of nonmetal materials has been used to support the system with stability by eliminating the vibration sources [2]. The whole system works continuously and steadily in the rigorous electromagnetic environment during the experiment. The line averaged electron density has been measured, ranging from $10^{18} \mathrm{~m}^{-3}$ to $10^{19} \mathrm{~m}^{-3}$ in three different operation conditions on KTX. The spectrum of the averaged electron density fluctuation is also analyzed and compared with the magnetic signal to show a mode rotation with frequency around $4 \mathrm{kHz}$.

As shown in fig. 1, KTX is a middle size reversed field pinch device with a major radius $1.4 \mathrm{~m}$ and a minor radius $0.4 \mathrm{~m}$. The whole interferometer is placed very close to KTX device to make it a compact system. The ohmic heating coils with air core are adopted, so the stray magnetic field around the machine is strong. Between the yellow ohmic field coils, the maximum stray field is around 200 Gauss. On the other hand, the high loop voltage during the plasma current ramp phase means the whole system is surrounded by high stray electric field. The terahertz solid state source is protected by $5 \mathrm{~mm}$ thick aluminum boxes, which are covered by $1 \mathrm{~mm}$ thick permalloy shell for low frequency magnetic field shielding. Two sources are used to form a heterodyne interferometer [3]. One is fixed frequency $650 \mathrm{GHz}$ and the other is tunable from $630 \mathrm{GHz}$ to $655 \mathrm{GHz}$. The output power of each source is about $2 \mathrm{~mW}$ and the signals are received by mixers with sensitivity about $750 \mathrm{~mW} / \mathrm{mV}$. Epoxy resin are used as the major material of the platform to avoid eddy current and to make the system electronically isolated. The total epoxy resin boards weigh about

$200 \mathrm{~kg}$. Dominate vibration effects caused by eddy current have been suppressed to the same level as background phase noise, which is about $0.05 \mathrm{pi}$, corresponding to a line integral density measurement accuracy about $10^{17} \mathrm{~m}^{-2}$. The spatial resolution is $3 \mathrm{~cm}$ in the radial direction, which is constraint by the probing beam width through plasma.

After the installation of the interferometer system, efforts were spent on the signal optimization further. The first problem is the refraction effect on signal amplitude received at detectors. As shown in fig. 2, the IF amplitude detected at the mixer almost dropped to zero during the discharge, while the reference signal was not affected at all. This phenomenon indicates that the probing beam deviated from the receive port of the Schottky diode mixer during the discharge, which is probably caused by beam refraction through plasma. The width of the mixer input port is about $4 \mathrm{~mm}$. The KTX vacuum port is nar- row with a diameter only $35 \mathrm{~mm}$. Those restrictions result in strict condition of beam alignment. After carefully aligning the centers of probing beam and the vacuum window, the 'zero amplitude' phenomenon disappeared.

The second problem is the vibration problem.

As phase measurement system, the interferometer can provide an accurate estimation of the vibration level. Fig. 3 show the phase shift caused by system vibration during the discharge without plasma. The gray shading area is the time period of plasma pulse. During this period the vibration amplitude is less than $0.1 \pi$, which is consistent with previous bench test results [2]. By analyzing the spectra of the phase vibration signals, it is inferred that the initial vibration between $12 \mathrm{~ms}$ and $30 \mathrm{~ms}$ is the vibration of the resin board close to the vacuum window between two toroidal coils. And the late low frequency is the vibration of the support pillar. Since there is no direct contact between the whole system and the KTX machine, the vibration is caused by the sound wave propagation. In a word, the density measurement is not effected by the current vibration level. When the plasma pulse length is optimized to about $100 \mathrm{~ms}$ after the coming KTX upgrade, this problem require to be take into consideration. Another pillar may need to strength the support structure.

Line integrated density is provided by the phase difference between the reference IF signal and the measurement IF signal. The intermediate frequency is set at $2.3 \mathrm{MHz}$, corresponding to a time resolution about $0.4 \mu \mathrm{s}$. Here a digital phase comparator technique [4] is used instead of analog phase comparator. KTX is now in conditioning phase, and could be operated in three different mode: Short pulse Tokamak discharge with high current (TS), Long pulse Tokamak discharge with low current (TL) and RFP discharge. In TS mode the maximum plasma current can research $200 \mathrm{kA}$. However since the maximum toroidal field of KTX is only 0.2 Tesla, this low q configuration is unstable and the pulse duration is only about $9 \mathrm{~ms}$. In TL mode the maximum plasma current can research $50 \mathrm{kA}$. The pulse duration can research $25 \mathrm{~ms}$, which is already beyond the shell penetration time. The minimum edge $\mathrm{q}$ is around 2.8 in this configuration, which is more stable than that in TS mode. The maximum plasma current in RFP mode is about $70 \mathrm{kA}$ and the discharge time is about $5 \mathrm{~ms}$. In RFP configuration, multi-tearing mode tent to resonant at different radial positions and overlap with each other [5]. That deteriorates the confinement and increases the plasma resistivity.

Figure 4 shows the waveforms of plasma current and center chord averaged electron density in TS, TL and RFP discharge mode. The density fluctuation amplitudes are larger in TS and RFP compared with that in TL condition. This is consistent with the stability condition mentioned above. In TS and RFP mode, plasma is more likely 
to scrape the wall, which results in severe neutral recycling. Another possibility is that the particle flux induced from poor confinement hit the wall and cause impurity sputtering out. The density operation region is also provided by the interferometer as shown in fig 5 . The chord averaged density range from $0.5 \times 10^{19} \mathrm{~m}^{-3}$ to $3 \times 10^{19} \mathrm{~m}^{-3}$.

Figure 6 shows the comparison between spectrum of line electron density fluctuation and spectrum of edge magnetic fluctuation field. It is clearly shown in fig. 6a) the amplitude of fluctuation is about $0.05 \times 10^{19} \mathrm{~m}^{-3}$. Both spectrum of density fluctuation and magnetic fluctuation indicate that a mode with frequency around $4 \mathrm{kHz}$ exists during the whole discharge time, and the mode should be electromagnetic. It also can be inferred that the poloidal mode numbers should be odd because the line integrated diagnostics cannot detect the fluctuations with even mode number. This is consistent with the mode number calculation based on poloidal mirnov array signal using SVD method. Above all, all results show the successful application of terahertz solid source based interferometer system to measure weak density fluctuation on toroidal magnetic confinement fusion device.

This work is supported by the Fundamental Research Funds for the Central Universities and the National Magnetic Confinement Fusion Energy Program of China under Contract Nos. 2014GB109002. This work is also supported by the National Natural Science Foundation of China 11605199.

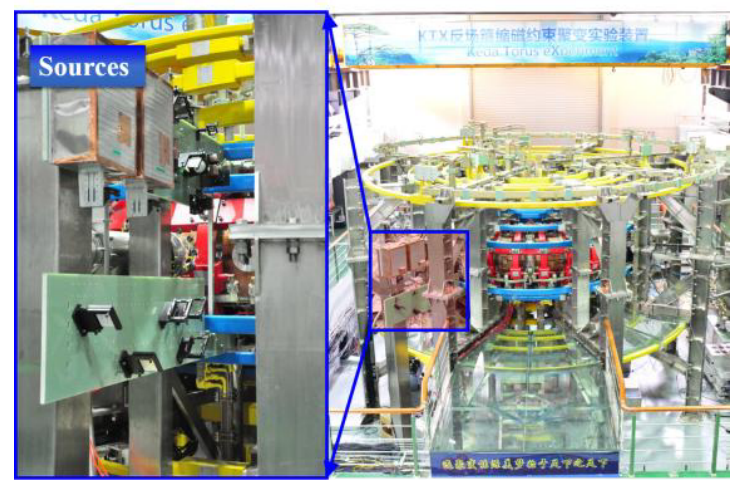

Fig. 1. Interferometer system arrangement on KTX device

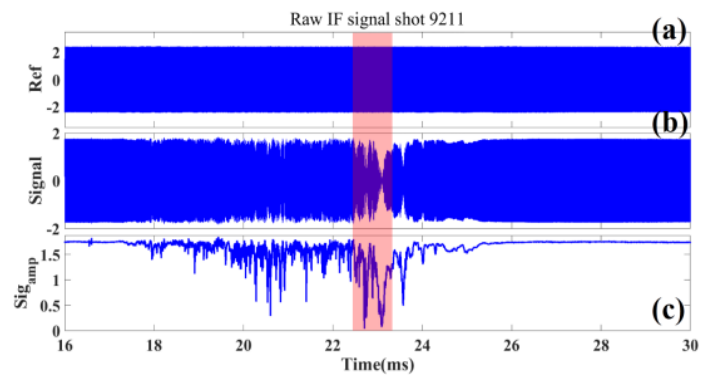

Fig. 2. Raw intermediate frequency (IF) signals: (a) reference signal, (b) probing beam signal and (c) envelop curve of diagnostic signal

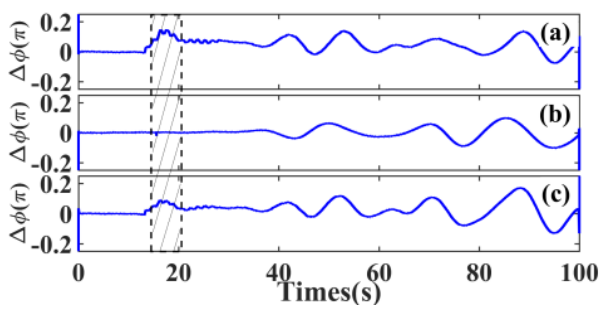

Fig. 3. Phase vibration in: a) toroidal field coils discharge only, b) Ohmic field coils discharge only, and c) Ohmic field coils and toroidal field coils discharge together

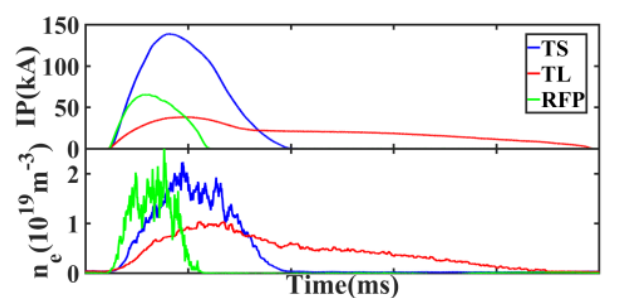

Fig. 4. Waveforms of plasma current and center chord averaged electron density in TS, TL and RFP condition

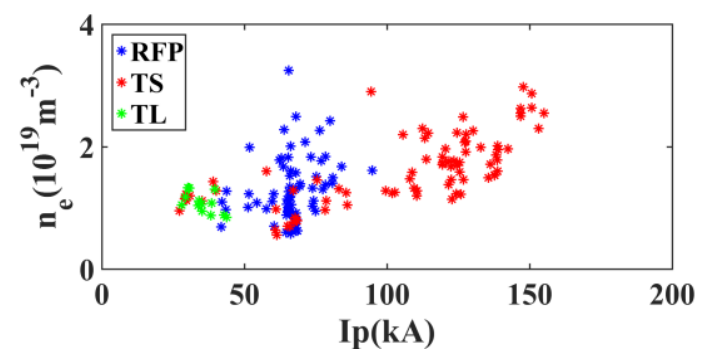

Fig. 5. Maximum plasma current versus corresponding chord averaged electron density

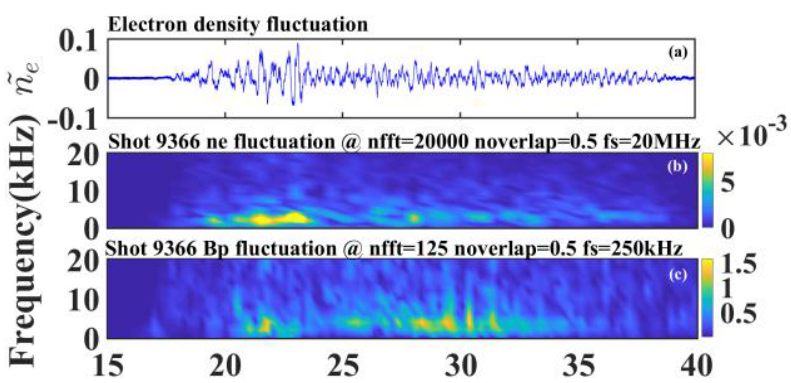

Fig. 6. a) Central chord averaged electron density fluctuation, b) Spectrum of averaged electron density fluctuation, c) Spectrum of mirnov probe

\section{References}

1. W. Liu et al., Plasma Phys. Control. Fusion. 56, 094009

2. Mao W et al. Rev. Sci. Instrum. 87, 11E122(2016).

3. J.L. Xie et al. Rev. Sci. Instrum. 85, 11D828(2014).

4. Y. Jiang et al. Rev. Sci. Instrum. 68, 902(1997).

5. P. Franz et al. Phys. Rev. Lett. 92, 125001.(2004). 